\section{The human PAF complex coordinates transcription with events downstream of RNA synthesis}

\author{
Bing Zhu, ${ }^{1}$ Subhrangsu S. Mandal, ${ }^{1}$ \\ Anh-Dung Pham, ${ }^{1}$ Yong Zheng, ${ }^{1}$ \\ Hediye Erdjument-Bromage, ${ }^{2}$ Surinder K. Batra, ${ }^{3}$ \\ Paul Tempst, ${ }^{2}$ and Danny Reinberg ${ }^{1,4}$ \\ ${ }^{1}$ Howard Hughes Medical Institute, Division of Nucleic Acids \\ Enzymology, Department of Biochemistry, University of \\ Medicine and Dentistry of New Jersey, Robert Wood Johnson \\ Medical School, Piscataway, New Jersey 08854, USA; \\ ${ }^{2}$ Molecular Biology Program, Memorial Sloan Kettering \\ Cancer Center, New York, New York 10021, USA; \\ ${ }^{3}$ Department of Biochemistry and Molecular Biology, \\ University of Nebraska Medical Center, \\ Omaha, Nebraska 68198-4525, USA
}

The yeast PAF (yPAF) complex interacts with RNA polymerase II and coordinates the setting of histone marks associated with active transcription. We report the isolation and functional characterization of the human PAF (hPAF) complex. hPAF shares four subunits with yPAF (hCtr9, hPaf1, hLeo1, and hCdc73), but contains a novel higher eukaryotic-specific subunit, hSki8. RNAi against hSki8 or hCtr9 reduces the cellular levels of other hPAF subunits and of mono- and trimethylated H3-Lys 4 and dimethylated H3-Lys 79. The hSki8 subunit is also a component of the human SKI (hSKI) complex. Yeast SKI complex is cytoplasmic and together with Exosome mediates 3 '-5' mRNA degradation. However, hSKI complex localizes to both nucleus and cytoplasm. Immunoprecipitation experiments revealed that hPAF and hSKI complexes interact, and ChIP experiments demonstrated that hSKI associates with transcriptionally active genes dependent on the presence of hPAF. Thus, in addition to coordinating events during transcription (initiation, promoter clearance, and elongation), hPAF also coordinates events in RNA quality control.

Supplemental material is available at http://www.genesdev.org.

Received December 20, 2004; revised version accepted May 27, 2005.

The PAF complex was first identified in yeast (yPAF) as an RNA polymerase II-associated factor, consisting of five subunits, Paf1, Ctr9, Leo1, Rtf1, and Cdc73 (Shi et al. 1996; Krogan et al. 2002; Mueller and Jaehning 2002). The yPAF complex interacts with TBP (Stolinski et al. 1997), the elongation factors Spt4-Spt5, and FACT

[Keywords: PAF; RNA surveillance; SKI; transcription]

${ }^{4}$ Corresponding author.

E-MAIL reinbedf@umdnj.edu; FAX (732) 235-5294.

Article and publication are at http://www.genesdev.org/cgi/doi/10.1101/ gad.1292105.
(Costa and Arndt 2000; Krogan et al. 2002; Squazzo et al. 2002). Chromatin immunoprecipitation (ChIP) experiments revealed the presence of the yPAF complex at both promoter and coding regions of transcriptionally active genes (Pokholok et al. 2002). Therefore, yPAF was postulated to be involved in both RNA polymerase IImediated transcription initiation and elongation. Recently, the yPAF complex has been shown to be required for Rad6-mediated histone H2B-K123 monoubiquitination (Ng et al. 2003b; Wood et al. 2003), Set1-mediated histone H3-K4 methylation (Krogan et al. 2003; Ng et al. 2003a), and Dot1-mediated histone H3-K79 methylation (Krogan et al. 2003), placing PAF at the center of cotranscriptional histone modifications (for review, see Hampsey and Reinberg 2003). PAF subunits are also required for maintaining proper poly(A) tail length in yeast, suggesting a post-transcriptional role for PAF complex in mRNA processing and maturation (Mueller et al. 2004).

Cotranscriptional events include not only chromatin modifications but also RNA processing and surveillance. Cotranscriptional RNA processing events such as $5^{\prime}$-end capping, splicing, and polyadenylation are relatively well documented (for reviews, see Maniatis and Reed 2002; Neugebauer 2002; Orphanides and Reinberg 2002). In recent years, evidence for cotranscriptional RNA surveillance, particularly in higher eukaryotes, emerged as well. Higher eukaryotes developed the process of alternative splicing to generate multiple mRNAs from one gene. This requires RNA surveillance to ensure high fidelity of gene expression. In mammals, nonsense-mediated decay (NMD) occurs not only in the cytoplasm, but also in the nucleus (Humphries et al. 1984; Takeshita et al. 1984; Cheng and Maquat 1993; Maniatis and Reed 2002). More recently, precursor RNAs containing nonsense codons were found to accumulate near the site of transcription in mammals (Muhlemann et al. 2001), suggesting that surveillance occurs around this region. A multi-subunit complex required for RNA 3 ' -5 ' mRNA decay, designated the Exosome, was also reported to be associated with transcriptionally active genes via its interaction with the transcription elongation factor Spt6 in Drosophila (Andrulis et al. 2002).

In this study we purified the human PAF (hPAF) complex. hPAF differs from yPAF in that it contains a novel higher-eukaryotic-specific subunit, hSki8. hSki8 is also a component of the human SKI (hSKI) complex. The yeast SKI complex and Exosome are both genetically required for 3'-to-5' mRNA decay (Ridley et al. 1984; Masison et al. 1995; Anderson and Parker 1998; Brown et al. 2000; Araki et al. 2001; Mitchell and Tollervey 2003). We found that hSKI localizes to transcriptionally active genes in an hPAF-dependent manner. This novel link between hPAF and hSKI supports the hypothesis that the hPAF complex coordinates events downstream of RNA synthesis such as RNA surveillance.

\section{Results and Discussion}

hPAF complex contains a novel higher-eukaryotic-specific subunit, hSki8

A cDNA encoding hPaf1 was originally isolated as PD2, a gene that is amplified and overexpressed in human pancreatic adenocarcinoma (S.K. Batra, unpubl.). We frac- 
tionated nuclear extracts derived from HeLa cells through conventional chromatography following the elution of hPaf1/Pd2 using antibody directed against Pd2 in Western analyses. The polypeptides coeluting with hPaf1 were identified by MALDI-TOF mass spectrometry. Besides hPaf1, three other human homologs of the yPAF complex were identified. Accordingly, they were termed hCtr9, hLeo1, and hCdc73 (Supplementary Figs. $1,2)$. In addition to these homologs of yPAF subunits, hSki8 also coelutes with the hPAF complex (see below).

Interestingly, no human homolog of the yeast Rtf1 was identified within the polypeptides coeluting with the hPAF complex during conventional purification (Supplementary Fig. 1). This was confirmed when hPAF complex was isolated from HEK-293F cells stably transfected with plasmids expressing either Flag-tagged hPafl or Flag-tagged hLeol. Nuclear extracts from these stable cell lines were subjected to affinity purification using M2 anti-Flag resin and then subjected to SDS-PAGE and MALDI-TOF mass spectrometry analyses. Affinity purification of Flag-hPafl or Flag-hLeol resulted in complexes exhibiting the same subunit composition as native purified hPAF: hCtr9, hLeo1, hPaf1, hCdc73, and hSki8 (Fig. 1A). These polypeptides were confirmed by Western analysis (Supplementary Fig. 3A). The affinitypurified hPAF complex was analyzed by fractionation on a gel filtration column. Silver staining of an SDS-polyacrylamide gel demonstrates five subunits-hCtr9,
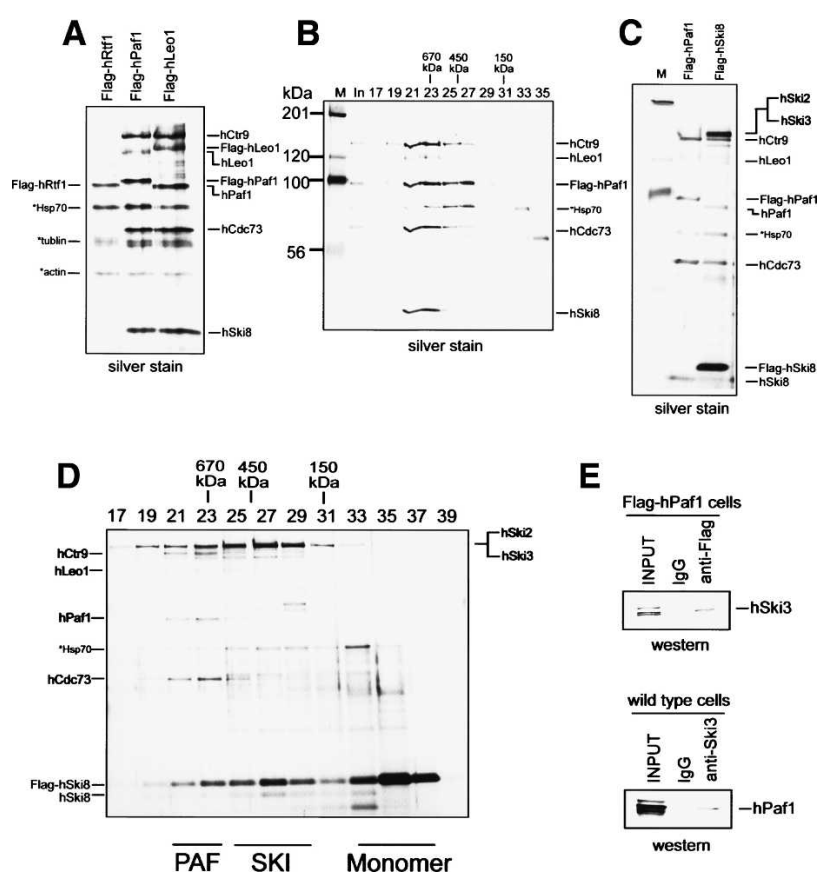

Figure 1. hSki8 is a novel higher-eukaryote-specific subunit of the hPAF complex, a subunit shared by the hSKI complex, which interacts with hPAF. (A) Silver stain of the affinity-purified hRtfl and hPAF complex. (B) Silver stain of the affinity-purified human PAF complex. Fractions were from a 2-mL Smart Superose 6 gel filtration column. The fraction numbers are shown on top of the panel. $(C)$ Silver stain of affinity-purified Flag-hPaf1 and Flag-hSki8. $(D)$ Silver stain of the affinity-purified Flag-hSki8. Fractions were from a 2-ml Smart Superose 6 gel filtration column. (E) Coimmunoprecipitation for interaction between hPAF and hSKI complexes. Asterisks indicate commonly contaminating, nonspecific proteins such as Hsp70, $\alpha$-tubulin, and actin.
hLeo1, hPaf1, hCdc73, and hSki8-coeluting as an 600kDa complex (Fig. 1B).

Neither conventional nor affinity-purified hPAF complex contained the Rtf1 homolog. However, an apparent human Rtfl homolog exists in the database, which shares $21 \%$ sequence identity and $40 \%$ similarity with the yeast Rtf1 (Supplementary Fig. 2). We designated this protein as hRtf1 and established a stable cell line expressing Flag-hRtf1. Affinity-purified hRtf1 did not display any subunits of the hPAF complex (Fig. 1A; Supplementary Fig. 3A). These results demonstrate that unlike in yeast, hRtf1 is not a subunit of the hPAF core complex. Fractionation of affinity-purified hRtf1 on a gel filtration column suggested that hRtfl has a native mass of $\sim 200 \mathrm{kDa}$ and it did not coelute with any other polypeptide (Supplementary Fig. 3B).

Our results are consistent with the studies of Rozenblatt-Rosen et al. (2005) demonstrating the existence of hCtr9, hLeo1, hPaf1, and hCde73 as hPAF subunits and the apparent loss of hRtfl as an integral subunit to the complex. However, our results identified hSki8 as a novel, higher-eukaryotic-specific subunit of the hPAF complex. We believe the difference is likely due to the purification approaches. In their report, hPAF was affinity-purified with antibodies against hCdc 73 and eluted with acidic glycine. This procedure inevitably elutes large amounts of IgG that likely masked the low-molecular-weight region of the SDS/PAGE gel. This would not impinge on the identification of subunits larger than IgG, but may have obscured the presence of hSki8, the only hPAF subunit smaller than the IgG heavy chain.

Since the yPAF complex includes Rtf1 and interacts with RNA polymerase II, we analyzed whether the hPAF complex and hRtfl interact with RNA polymerase II. Immunoprecipitation using antibody against hPafl specifically enriched for the subunits of the hPAF core complex, such as hCtr9, hLeo1, and hCdc73 as well as substoichiometric amounts of RNA polymerase II and hRtf1. Similarly, antibodies against RNA polymerase II and hRtf1 coimmunoprecipitated substoichiometric amounts of each other as well as subunits of the hPAF core complex (Supplementary Fig. 4). These results establish interactions between RNA polymerase II, the hPAF core complex, and hRtf1.

\section{hSki8 is also a subunit of the hSKI complex, and hPAF interacts with hSKI in vivo}

Little is known about hSki8; it is denoted after the yeast homolog Ski8. Yeast Ski8, together with Ski2 and Ski3, form a trimeric complex that is required for $3^{\prime}$-to-5' mRNA decay (Ridley et al. 1984; Masison et al. 1995; Brown et al. 2000). To further analyze the association of hSki8 with the hPAF complex, we established a stable cell line expressing Flag-hSki8. Affinity purification from the stable Flag-hSki8 cell line revealed the presence of the entire hPAF complex, further confirming hSki8 as an integral subunit (Fig. 1C). Interestingly, distinct higher-molecular-weight polypeptides were observed, apparently specific to the Flag-hSki8 complex, as they were absent in the purified Flag-hPafl and Flag-hLeo1 complexes (Fig. 1A,C). MALDI-TOF mass spectrometry analysis identified all the hPAF subunits as well as the homologs of the yeast Ski2 and Ski3 proteins (for sequence alignments, see Supplementary Fig. 2). There- 

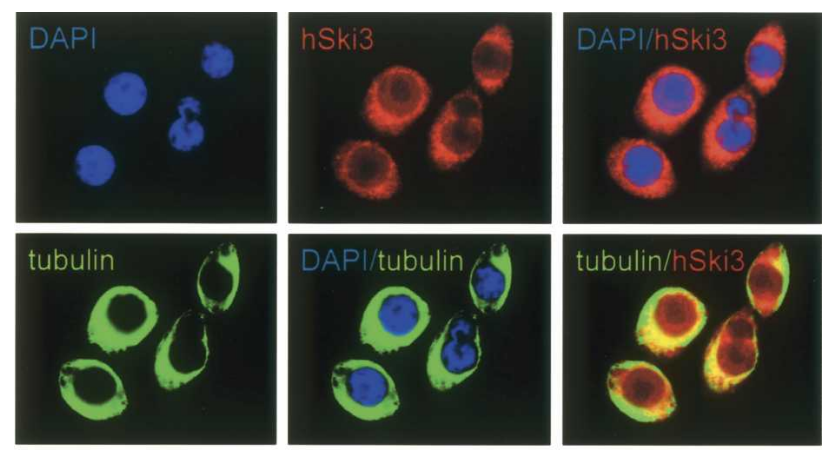

competed with recombinant hSki3
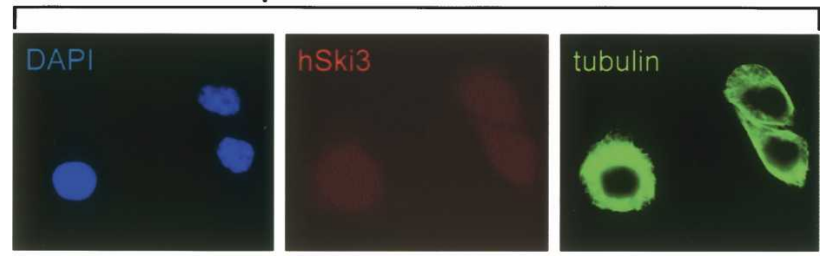

competed with recombinant hPaf1
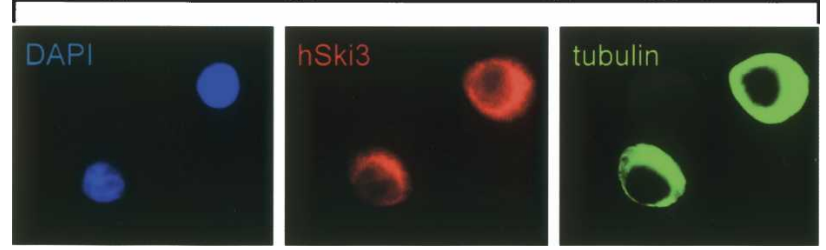

Figure 2. hSKI complex localizes to both cytoplasm and nucleus of HeLa cells. The upper panel shows staining of DAPI, hSki3, and $\alpha$-tubulin with corresponding merges. The lower panel shows the competition experiment with $10 \mu \mathrm{g}$ of purified recombinant proteins Ski3 or Paf1.

fore, we designated the high-molecular-weight proteins as hSki2 and hSki3. Gel filtration of the affinity-purified hSki8 sample revealed two distinct complexes, one corresponding to the hPAF complex and the other exhibiting the coelution of three polypeptides: hSki2, hSki3, and hSki8 (Fig. 1D). We denoted this second complex as hSKI. Under stringent conditions $(0.5 \mathrm{M} \mathrm{KCl})$ and using antibodies against hPaf1, we were able to deplete the hPAF complex from the affinity-purified Flag-hSki8. A gel filtration column revealed that the hPAF-depleted material contained a trimeric complex composed of hSki2, hSki3, and hSki8 eluting with an apparent native mass of $400 \mathrm{kDa}$ (Supplementary Fig. 3C).

The discovery of a novel subunit of the hPAF complex that is shared with the hSKI complex prompted us to investigate whether these two complexes interact. We performed immunoprecipitation experiments using nuclear extract derived from the Flag-hPaf1 stable cell line with antibodies against Flag. The results demonstrated substoichiometric amounts of hSki3 immunoprecipitated by anti-Flag antibody. Interestingly, whereas the extract (input) displayed apparently different isoforms of hSki3, the immunoprecipitated material contained only one isoform (Fig. 1E). Importantly, a reciprocal immunoprecipitation experiment using antibodies against hSki3 demonstrated immunoprecipitation of endogenous hPafl from nuclear extract (Fig. 1E). Thus, hPAF and hSKI complexes do interact.

\section{hSKI complex localizes to both cytoplasm and nucleus}

Yeast Ski2, Ski3, and Ski8, all subunits of the SKI complex, are essential for 3'-to-5' mRNA decay (Ridley et al. 1984; Masison et al. 1995). Additionally, the ySKI complex interacts with the Exosome, a multi-subunit complex essential for $3^{\prime}-5^{\prime}$ mRNA decay (Anderson and Parker 1998; Araki et al. 2001; Mitchell and Tollervey 2003). In Drosophila, an Exosome complex was found to be associated with transcriptionally active genes via its interaction with the transcription elongation factor Spt6, thus highlighting its role in cotranscriptional RNA surveillance (Andrulis et al. 2002). These studies, together with our findings that the hSKI complex, which may also participate in RNA decay, interacts with the hPAF complex involved in transcription and that they share a common subunit, prompted us to hypothesize that hSKI complex might also participate in cotranscriptional RNA surveillance.

The Exosome localizes to both the cytoplasm and the nucleus in yeast and mammals (Bousquet-Antonelli et al. 2000), whereas the SKI complex appears to be cytoplasmic in yeast (Brown et al. 2000). We next investigated the cellular localization of the hSKI complex. We performed immunofluorescence experiments to visualize the endogenous hSKI complex in HeLa cells using highly specific antibodies raised against hSki3. Interestingly, although hSki3 signal was detected in the cyto-

A
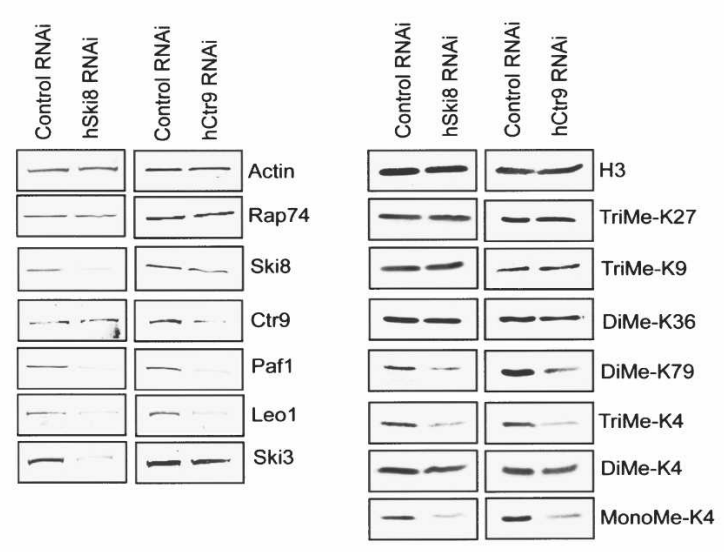

B

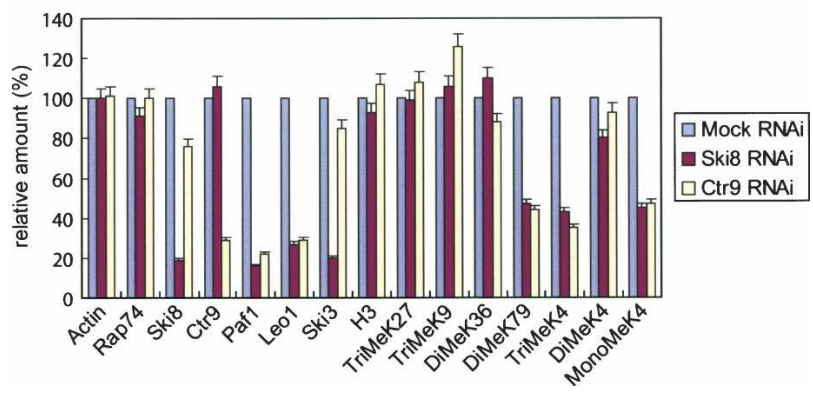

Figure 3. RNAi targeting hSki8 or hCtr9 knocks down the protein levels of other hPAF subunits and reduces cellular levels of histone H3-K4 trimethylation, monomethylation, and H3-K79 dimethylation. $(A)$ Western blots for various proteins and histone modifications with whole cell lysate from mock RNAi, hSki8 RNAi, and hCtr9 RNAi samples. (B) Quantification of the Western blots. 
plasm, a significant amount of hSki3 signal was also localized within the nucleus; we also noticed that the hSki3 signal appears to be excluded from the nucleolus (Fig. 2). As a control we used antibodies against $\alpha$-tubulin, which localizes exclusively to the cytoplasm (Fig. 2). Importantly, preincubation of the hSki3 antibodies with excess recombinant hSki3 protein, but not recombinant hPaf1 protein, inhibited the signal for hSki3, demonstrating that the hSki3 immunofluorescence is specific (Fig. 2, lower panels). In addition, hSki8 localized to both the cytoplasm and nucleus, while hPafl appears to be predominantly nuclear with a weak signal in the cytoplasm (Supplementary Fig. 5). This suggested that the hSKI complex may associate with transcriptionally active genes (see below) and mediate RNA surveillance.

\section{hSki8 is required for PAF-dependent mono- \\ and trimethylation of histone H3-K4 \\ and dimethylation of H3-K79 in vivo}

To understand the functional importance of the hPAF complex in vivo, we used RNAi against two of the hPAF subunits, hCtr9 and hSki8. Interestingly, RNAi directed against either hCtr9 or hSki8 not only knocked down the levels of the target proteins, but also other subunits of the hPAF complex such as hPafl and hLeol. As controls, the levels of actin and the subunit of the TFIIF transcription factor Rap74 were unaffected (Fig. 3). These results are consistent with another report showing that deletion of individual yPAF subunits leads to various degrees of reduction in the protein levels of the other subunits (Mueller et al. 2004).

We then investigated the histone methylation marks. In line with previous studies in yeast, RNAi against hCtr9 or hSki8 led to a significant reduction in the cellular levels of trimethylation of histone H3-K4 and dimethylation of histone $\mathrm{H} 3-\mathrm{K} 79$, but not in dimethylation of H3-K36. As controls, the total levels of histone H3 and methylation marks associated with repression (trimethylation of $\mathrm{H} 3-\mathrm{K} 9$ and $\mathrm{H} 3-\mathrm{K} 27$ ) were not affected. Interestingly, when the status of the different methylation marks for H3-K4 were analyzed, we found no significant reduction in dimethylation, but significant reductions in mono- and trimethylation (Fig. 3). This is probably due to the existence of multiple histone H3-K4 methyltransferases in humans (for review, see Margueron et al. 2005) and the distinct roles for the H3-K4 methylation marks. Trimethylated $\mathrm{H} 3-\mathrm{K} 4$ is well documented to be associated with transcription (Santos-Rosa et al. 2002; Schneider et al. 2004), but little is known about H3-K4 monomethylation. Our results suggest that histone H3-K4 monomethylation may also be a mark associated with active transcription.

hSKI complex is present at transcriptionally active genes with recruitment dependent on hPAF complex

Given the nuclear localization of a fraction of the hSKI complex and the physical interaction between hSKI and hPAF complexes, we next analyzed if the hSKI complex, likely a part of the RNA decay machinery, may function as a transcription-related factor. ChIP experiments using primers directed to the promoter and the downstream regions of the RPB1 gene demonstrated that the hSKI complex subunits (hSki3 and hSki8) are present at both the promoter and coding regions, generating a pattern similar to that observed for the hPAF complex subunits (hPaf1, hLeo1, and hSki8) (Fig. 4A). Importantly, while siRNA against the hPAF subunit hCtr9 did not affect hSki3 or hSki8 protein levels (Fig. 3), the ChIP signals for hSKI and hPAF were both similarly reduced. On the other hand, TFIIB and RNA polymerase II (Rpb4) signals remained the same (Fig. 4A).

To further analyze the presence of the human SKI complex at transcriptionally active genes, the inducible $M A G E-A 1$ gene was chosen. The expression of the MAGE gene family (melanoma antigen genes) is regulated by DNA methylation. These genes are heavily methylated and are transcriptionally inactive in most human tissues and cell lines, while the DNA demethylating agent 5-aza-deoxycytidine (5-aza-dC) induces the expression of MAGE genes in various cells (De Smet et al. 1996). In the HEK293 cells used in this study, the $M A G E-A 1$ gene is also known to be methylated and silent (Fig. 4B; B. Zhu and J.P. Jost, unpubl.). Expression of the MAGE-A1 gene was observed after $48 \mathrm{~h}$ of 5 -aza-dC treatment (Fig. 4B). Like hPAF complex, hSKI was absent at the methylated and transcriptionally inert $M A G E-A 1$ gene. However, upon induction, hSKI was recruited to the promoter and coding sequences of the MAGE-A1 gene, along with other factors of the transcription machinery. This hSKI recruitment was again reduced after knock-down of the hPAF complex by siRNA targeting hCtr9 (Fig. 4B).

These data collectively suggest that hSKI, a protein complex that is required for $3^{\prime}$-to- $5^{\prime}$ RNA decay in yeast (Masison et al. 1995; Brown et al. 2000), associates with transcriptionally active genes via its interaction with the hPAF complex in the case of human cells. Notably, Exosome, the other complex that is required for RNA $3^{\prime}-5^{\prime}$ decay, was also reported to be associated with transcriptionally active genes via its interaction with the transcription elongation factor Spt6 (Andrulis et al. 2002).
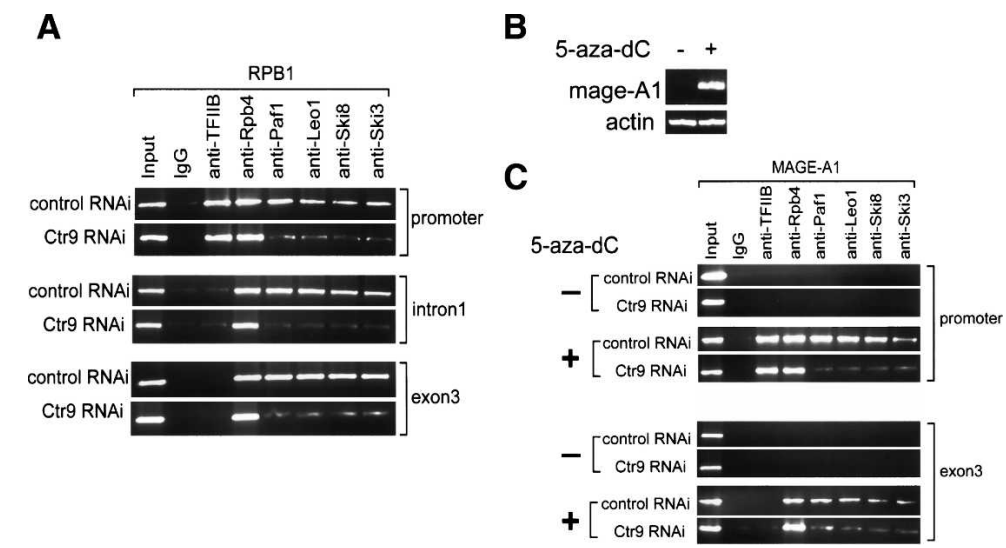

Figure 4. hSKI complex is present at transcriptionally active genes, and its recruitment is dependent on hPAF. (A) Human SKI complex is present at promoter and coding regions of the $R P B 1$ gene and hPAF knock-down reduces the signal for hSKI complex. (B) RT-PCR showing the induction of MAGEA1 expression upon 5-aza-dC treatment. (C) Human SKI complex is recruited to the $M A G E-A 1$ gene upon its induction, together with the RNA polymerase II transcription machinery. hSKI recruitment is hPAF-complex-dependent. 
Interestingly, Exosome occupies both the promoter and coding regions as well (Andrulis et al. 2002). Taken together, the human SKI complex shares many common features with the Exosome complex as both likely compose the 3 ' -5 ' RNA decay machinery in higher eukaryotes, interact with transcription factors, and occupy transcriptionally active genes. Therefore, we suggest that the hSKI complex, together with the Exosome, likely regulate cotranscriptional events including mRNA quality control. That a portion of hSKI complex is present in the nucleus and that human-derived SKI and PAF complexes interact are distinct differences with the case in yeast. This may signify a novel additional function(s) for hPAF and hSKI complexes or that higher eukaryotes evolved to integrate transcription and surveillance more efficiently.

\section{The PAF complex: elongation factor or modulator of RNA metabolism?}

Several findings have demonstrated interactions between transcription elongation factors and either the yeast (Costa and Arndt 2000; Krogan et al. 2002; Squazzo et al. 2002) or the human PAF complexes (B. Zhu and D. Reinberg, unpubl.). Yet, Jaehning and coworkers found that the loss of the PAF complex in yeast does not alter the distribution of RNA polymerase II or transcription elongation factors such as Spt5 and Spt16 on transcriptionally active genes (Mueller et al. 2004). Moreover, although the PAF complex was detected in association with randomly selected active yeast genes (Pokholok et al. 2002), suggesting it has a general role in transcription, deletion of the PAF complex (or its subunits) in yeast and plants affected the expression of only a subset of genes (Porter et al. 2002; He et al. 2004; Oh et al. 2004). These observations collectively suggest that the PAF complex does not function as a classical elongation factor, despite the fact that PAF is associated with the transcriptionally active RNA polymerase II (Mueller et al. 2004; Sims et al. 2004). Our results and the previously published results with Exosome support the model that mRNA surveillance occurs "cotranscriptionally" rather than "posttranscriptionally." We favor the hypothesis that the hPAF complex serves as a platform between RNA polymerase II and many other factors modulating RNA biogenesis, in essence modulating cotranscriptional events such as chromatin remodeling, chromatin modifications, mRNA maturation, and surveillance.

\section{Materials and methods}

GenBank accession numbers

The GenBank accession numbers are as follows: hPafl, AJ401156; hLeol, BC018147; hCtr9, BC058914; hCdc73, NM_024529; hSki8, AK024754; hRtf1, BC015052; hSki3, BC056893; and hSki2, Q15477.

Affinity purification of hRtf1 and the hPAF complex

M2 anti-Flag agarose (Sigma) was equilibrated with the same buffer used in nuclear extract preparation $(20 \mathrm{mM}$ Tris- $\mathrm{HCl}$ at $\mathrm{pH} 7.9,1.5 \mathrm{mM}$ $\mathrm{MgCl}_{2}, 0.42 \mathrm{M} \mathrm{NaCl}, 0.2 \mathrm{mM}$ PMSF) and then incubated with nuclear extract derived from various stable cell lines overnight at $4^{\circ} \mathrm{C}$. The resin was washed with an excess amount of buffer $\mathrm{C}$ containing $0.5 \mathrm{M} \mathrm{KCl}$ plus $0.1 \% \mathrm{NP}-40$, and then eluted with buffer C containing $0.5 \mathrm{M} \mathrm{KCl}$ plus 0.1 $\mathrm{mg} / \mathrm{mL}$ Flag peptide.

Coimmunoprecipitation

HeLa cell nuclear extracts were dialyzed against buffer C containing 0.1 $\mathrm{M} \mathrm{KCl}$, and then incubated with various antibodies cross-linked to pro- tein A agarose. The resin was washed with an excess amount of buffer C containing $0.15 \mathrm{M} \mathrm{KCl}$, and proteins were eluted with $0.1 \mathrm{M}$ glycine- $\mathrm{HCl}$ at $\mathrm{pH} 2.5$.

\section{ChIP}

ChIP was performed according to the protocol described by Upstate. For every assay, cells were derived from approximately one confluent $15-\mathrm{cm}$ plate.

RNAi knock-down for the hPAF complex

siRNAs targeting hSki8 and hCtr9 were purchased from Dharmacon. siRNAs were delivered using RNAiFect from QIAGEN according to the user manual.

\section{Immunofluorescence}

HeLa cells along with monoclonal antibody B-5-1-2 against $\alpha$-tubulin (Sigma) and affinity-purified rabbit polyclonal antibodies against hSki3, hSki8, and hPafl were used in the experiments.

\section{Acknowledgments}

We are grateful to Dr. Stephen Desiderio from the Department of Molecular Biology and Genetics, John Hopkins University School of Medicine, for kindly providing antibody against hCtr9/p150TSP. We thank the PGA antibody core center for biomedical inventions and University of Texas Southwestern Medical Center at Dallas for providing antibody against hCdc73. We thank Dr. Matthew Meyerson for communicating results before publication. We are gratefully to Dr. L.D. Vales for valuable comments on the manuscript. This study was supported by a grant from NIH (GM37120) and by the Howard Hughes Medical Institute (to D.R.) and by NCI Cancer Center Support Grant P30 CA08748 to P.T.

\section{References}

Anderson, J.S. and Parker, R.P. 1998. The $3^{\prime}$ to $5^{\prime}$ degradation of yeast mRNA is a general mechanism for mRNA turnover that requires the SKI2 DEVH box protein and $3^{\prime}$ to $5^{\prime}$ exonucleases of the exosome complex. EMBO J. 17: 1497-1506.

Andrulis, E.D., Werner, J., Nazarian, A., Erdjument-Bromage, H., Tempst, P., and Lis, J.T. 2002. The RNA processing exosome is linked to elongating RNA polymerase II in Drosophila. Nature 420: 837 841.

Araki, Y., Takahashi, S., Kobayashi, T., Kajiho, H., Hoshino, S., and Katada, T. 2001. Ski7p G protein interacts with the exosome and the Ski complex for $3^{\prime}$-to-5' mRNA decay in yeast. EMBO J. 20: 4684 4693.

Bousquet-Antonelli, C., Presutti, C., and Tollervey, D. 2000. Identification of a regulated pathway for nuclear pre-mRNA turnover. Cell 102: 765-775

Brown, J.T., Bai, X., and Johnson, A.W. 2000. The yeast antiviral proteins Ski2p, Ski3p, and Ski8p exist as a complex in vivo. RNA 6: 449-457.

Cheng, J. and Maquat, L.E. 1993. Nonsense codons can reduce the abundance of nuclear mRNA without affecting the abundance of premRNA or the half-life of cytoplasmic mRNA. Mol. Cell. Biol. 13: 1892-1902.

Costa, P.J. and Arndt, K.M. 2000. Synthetic lethal interactions suggest a role for the Saccharomyces cerevisiae Rtfl protein in transcription elongation. Genetics 156: 535-547.

De Smet, C., De Backer, O., Faraoni, I., Lurquin, C., Brasseur, F., and Boon, T. 1996. The activation of human gene MAGE-1 in tumor cells is correlated with genome-wide demethylation. Proc. Natl. Acad. Sci. 93: 7149-7153.

Hampsey, M. and Reinberg, D. 2003. Tails of intrigue: Phosphorylation of RNA polymerase II mediates histone methylation. Cell 113: 429-432.

He, Y., Doyle, M.R., and Amasino, R.M. 2004. PAF1-complex-mediated histone methylation of FLOWERING LOCUS C chromatin is required for the vernalization-responsive, winter-annual habit in Arabidopsis. Genes \& Dev. 18: 2774-2784.

Humphries, R.K., Ley, T.J., Anagnou, N.P., Baur, A.W., and Nienhuis, A.W. 1984. $\beta$ O-39 thalassemia gene: A premature termination codon causes $\beta$-mRNA deficiency without affecting cytoplasmic $\beta$-mRNA stability. Blood 64: 23-32. 
Krogan, N.J., Kim, M., Ahn, S.H., Zhong, G., Kobor, M.S., Cagney, G., Emili, A., Shilatifard, A., Buratowski, S., and Greenblatt, J.F. 2002. RNA polymerase II elongation factors of Saccharomyces cerevisiae: A targeted proteomics approach. Mol. Cell. Biol. 22: 6979-6992.

Krogan, N.J., Dover, J., Wood, A., Schneider, J., Heidt, J., Boateng, M.A., Dean, K., Ryan, O.W., Golshani, A., Johnston, M., et al. 2003. The Paf1 complex is required for histone H3 methylation by COMPASS and Dotlp: Linking transcriptional elongation to histone methylation. Mol. Cell 11: 721-729.

Maniatis, T. and Reed, R. 2002. An extensive network of coupling among gene expression machines. Nature 416: 499-506.

Margueron, R., Trojer, P., and Reinberg, D. 2005. The key to development: Interpreting the histone code? Curr. Opin. Genet. Dev. 15: 163-176.

Masison, D.C., Blanc, A., Ribas, J.C., Carroll, K., Sonenberg, N., and Wickner, R.B. 1995. Decoying the cap-mRNA degradation system by a double-stranded RNA virus and poly(A)-mRNA surveillance by a yeast antiviral system. Mol. Cell. Biol. 15: 2763-2771.

Mitchell, P. and Tollervey, D. 2003. An NMD pathway in yeast involving accelerated deadenylation and exosome-mediated $3^{\prime} \rightarrow 5^{\prime}$ degradation. Mol. Cell 11: 1405-1413.

Mueller, C.L. and Jaehning, J.A. 2002. Ctr9, Rtf1, and Leo1 are components of the Paf1/RNA polymerase II complex. Mol. Cell. Biol. 22: 1971-1980.

Mueller, C.L., Porter, S.E., Hoffman, M.G., and Jaehning, J.A. 2004. The Pafl complex has functions independent of actively transcribing RNA polymerase II. Mol. Cell 14: 447-456.

Muhlemann, O., Mock-Casagrande, C.S., Wang, J., Li, S., Custodio, N., Carmo-Fonseca, M., Wilkinson, M.F., and Moore, M.J. 2001. Precursor RNAs harboring nonsense codons accumulate near the site of transcription. Mol. Cell 8: 33-43.

Neugebauer, K.M. 2002. On the importance of being co-transcriptional. J. Cell Sci. 115: 3865-3871.

Ng, H.H., Robert, F., Young, R.A., and Struhl, K. 2003a. Targeted recruitment of Setl histone methylase by elongating Pol II provides a localized mark and memory of recent transcriptional activity. Mol. Cell 11: 709-719.

Ng, H.H., Dole, S., and Struhl, K. 2003b. The Rtf1 component of the Paf1 transcriptional elongation complex is required for ubiquitination of histone H2B. J. Biol. Chem. 278: 33625-33628.

Oh, S., Zhang, H., Ludwig, P., and van Nocker, S. 2004. A mechanism related to the yeast transcriptional regulator Paflc is required for expression of the Arabidopsis FLC/MAF MADS box gene family. Plant Cell 16: 2940-2953.

Orphanides, G. and Reinberg, D. 2002. A unified theory of gene expression. Cell 108: 439-451.

Pokholok, D.K., Hannett, N.M., and Young, R.A. 2002. Exchange of RNA polymerase II initiation and elongation factors during gene expression in vivo. Mol. Cell 9: 799-809.

Porter, S.E., Washburn, T.M., Chang, M., and Jaehning, J.A. 2002. The yeast pafl-rNA polymerase II complex is required for full expression of a subset of cell cycle-regulated genes. Eukaryot. Cell 1: 830-842.

Ridley, S.P., Sommer, S.S., and Wickner, R.B. 1984. Superkiller mutations in Saccharomyces cerevisiae suppress exclusion of M2 doublestranded RNA by L-A-HN and confer cold sensitivity in the presence of M and L-A-HN. Mol. Cell. Biol. 4: 761-770.

Rozenblatt-Rosen, O., Hughes, C.M., Nannepaga, S.J., Shanmugam, K.S., Copeland, T.D., Guszczynski, T., Resau, J.H., and Meyerson, M. 2005. The parafibromin tumor suppressor protein is part of a human Paf1 complex. Mol. Cell. Biol. 25: 612-620.

Santos-Rosa, H., Schneider, R., Bannister, A.J., Sherriff, J., Bernstein, B.E., Emre, N.C., Schreiber, S.L., Mellor, J., and Kouzarides, T. 2002. Active genes are tri-methylated at K4 of histone H3. Nature 419: 407411.

Schneider, R., Bannister, A.J., Myers, F.A., Thorne, A.W., Crane-Robinson, C., and Kouzarides, T. 2004. Histone H3 lysine 4 methylation patterns in higher eukaryotic genes. Nat. Cell Biol. 6: 73-77.

Shi, X., Finkelstein, A., Wolf, A.J., Wade, P.A., Burton, Z.F., and Jaehning, J.A. 1996. Paflp, an RNA polymerase II-associated factor in Saccharomyces cerevisiae, may have both positive and negative roles in transcription. Mol. Cell. Biol. 16: 669-676.

Sims III, R.J., Belotserkovskaya, R., and Reinberg, D. 2004. Elongation by RNA polymerase II: The short and long of it. Genes \& Dev. 18: $2437-$
2468

Squazzo, S.L., Costa, P.J., Lindstrom, D.L., Kumer, K.E., Simic, R., Jennings, J.L., Link, A.J., Arndt, K.M., and Hartzog, G.A. 2002. The Paf1 complex physically and functionally associates with transcription elongation factors in vivo. $E M B O$ J. 21: 1764-1774.

Stolinski, L.A., Eisenmann, D.M., and Arndt, K.M. 1997. Identification of RTF1, a novel gene important for TATA site selection by TATA box-binding protein in Saccharomyces cerevisiae. Mol. Cell. Biol. 17: 4490-4500.

Takeshita, K., Forget, B.G., Scarpa, A., and Benz Jr., E.J. 1984. Intranuclear defect in $\beta$-globin mRNA accumulation due to a premature translation termination codon. Blood 64: 13-22.

Wood, A., Schneider, J., Dover, J., Johnston, M., and Shilatifard, A. 2003. The Pafl complex is essential for histone monoubiquitination by the Rad6-Brel complex, which signals for histone methylation by COMPASS and Dotlp. J. Biol. Chem. 278: 34739-34742. 


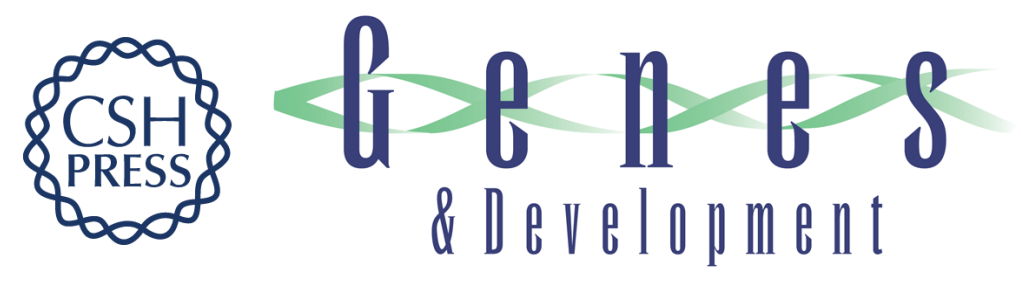

\section{The human PAF complex coordinates transcription with events downstream of RNA synthesis}

Bing Zhu, Subhrangsu S. Mandal, Anh-Dung Pham, et al.

Genes Dev. 2005, 19:

Access the most recent version at doi:10.1101/gad.1292105

\section{Supplemental http://genesdev.cshlp.org/content/suppl/2005/07/12/19.14.1668.DC1 Material}

References This article cites 37 articles, 23 of which can be accessed free at: http://genesdev.cshlp.org/content/19/14/1668.full.html\#ref-list-1

\section{License}

Email Alerting

Receive free email alerts when new articles cite this article - sign up in the box at the top Service

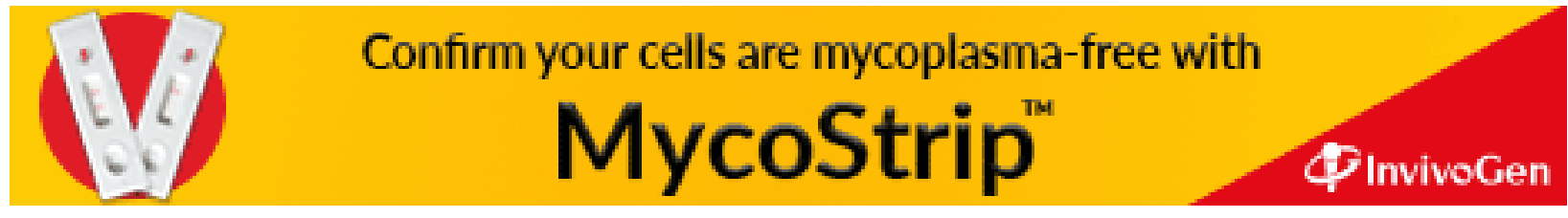

